\title{
Effect of Solvent Refractive Index on the Surface Plasmon Resonance Nanoparticle Optical Absorption
}

\author{
Gulay Ertas* and Sefik Suzer \\ Chemistry Department, Bilkent University, 06800 Ankara, Turkey
}

\begin{abstract}
Optical properties of plasmon coupled silver and gold nanoparticles were studied as a function of the refractive index of the surrounding medium. Our studies confirmed that the effect of changes in the refractive index of the surrounding medium was more difficult to demonstrate from an experimental point of view, because of the very high susceptibility of nanoparticles to aggregate in aqueous and organic solvents. Whereas the position of the absorption bands of triiodide in these solvents shows a clear dependence on medium's refractive index, the surface plasmon band position of silver and gold nanoparticles do not exhibit the same dependence. This is attributed to a non-negligible interaction of these solvents with nanoparticle surfaces.
\end{abstract}

Keywords: Gold and Silver Nanoparticles, Refractive Index.

\section{INTRODUCTION}

Synthesis of nanoparticles of metals in aqueous as well as in non-aqueous media by chemical, radiolytic and photocatalytic reactions and their optical properties have been of great interest over the last century. The optical response of noble metal nanoparticles is often characterized by the presence of a strong absorption band which is attributed to a resonance in the collective motion of the conduction electrons, in response to an incident electromagnetic fields, and is called as localized surface plasmon resonance (SPR). ${ }^{1}$ Frequency of these resonances depend on particle size, shape, composition, and optical properties of local environment in which the nanoparticles are immersed. ${ }^{1-7}$ The size effect has been experimentally studied for particles embedded in polymer films, ${ }^{8-11}$ partially oxidized Si matrix, ${ }^{12}$ and for silver clusters interacting with another metal, semiconductor or dielectric cluster. ${ }^{13}$ Solvent effects on the excitation spectra of periodic arrays of silver nanoclusters in water, ${ }^{14}$ fabricated by nanosphere lithography, ${ }^{4}$ modified with alkanethiol self assembly monolayers, ${ }^{15}$ and silica coated gold nanoparticles ${ }^{16,17}$ have also been thoroughly investigated. A brief overview of nanoparticles/ surrounding medium interaction was given by Kreibig and co-workers. ${ }^{18}$

The SPR plays an important role in various technological applications, ranging from chemical sensing of various modalities to cancer detection and therapy. ${ }^{19-22}$ Plasmon resonance based sensing can be accomplished in a variety

*Author to whom correspondence should be addressed. of ways and recently, localized metal nanostructures and nanopatterned surfaces have been used as nanoscale SPR sensors, both in solution and immobilized on surfaces. ${ }^{23,24}$ In these cases, it is claimed that changes in the plasmon resonance energy due to modifications in the refractive index of the surrounding medium are detected as wavelength shifts of the plasmon resonance spectrum for dispersed nanoparticles.

Although a number of groups have reported the absorption spectra of nanoparticles prepared in water and in organic solvents, comparison of the spectra is not easy because of differences in particle shape, size distribution, and colloidal stability. In order to eliminate this handicap, Underwood and Mulvaney ${ }^{25}$ have prepared gold nanoparticles in water and transferred into a variety of organic solvents using a "comb stabilizer," and claimed that this transformation occurred without causing coagulation and retaining the monodispersity of the original aqueous gold nanoparticles. This was stated through the observation that by allowing $\mathrm{CS}_{2}$ (solvent) to evaporate, the nanoparticles became redder again after redispersing in butyl acetate solvent as the refractive index decreased. In another study, the same group, Templeton et al., ${ }^{26}$ compared theory with experimental results for the effect of solvent refractive index and the core charge on the SPR band of solutions of alkanethiolate monolayer-protected gold clusters with an average core diameter of $5.2 \mathrm{~nm}$. Spectra of dodecanethiolate protected gold clusters revealed $8 \mathrm{~nm}$ red shift as the solvent refractive index was varied from 1.33 to 1.55 by transferring gold nanoparticles into appropriate solvents e.g., hexane, tetrahydrofuran, benzene etc. ${ }^{26}$ However, another study, ${ }^{27}$ with smaller diameter, unfractionated 
dodecanethiolate protected $\mathrm{Au}$ cluster $(4.4 \mathrm{~nm}$ average diameter), over a narrower solvent refractive index range (1.375-1.501), failed to detect this shift.

No further efforts were made to understand the mode of interaction of metal particle surfaces with the binding agents and solvent molecules in all of these studies. The complete dampening of the surface plasmon band of thiolcapped nanoparticles is an evidence of strong interaction between the metal surface and the organic binding agents. The loss of plasmon absorption in such systems limits the spectroscopic investigations on the influence of solvent environment on the surface of metal nanoparticles. In a different study carried out by Thomas et al., ${ }^{28}$ gold nanoparticles stabilized by tetraoctylammonium bromide (TOAB) were used to investigate the effect of refractive index on the surface plasmon absorption of Au nanoparticles instead of thiol capped nanoparticles, since TOAB capped particles exhibit spectroscopicly cleaner plasmon absorption bands when compared to thiol-capped ones. They observed a red shift in the SPR peak maximum with increase in solvent dielectric constant for solvents which do not complex with metal surface. In addition, they claimed that the surface plasmon band position remains unaffected in polar solvents with non-bonding electrons, which was attributed to the direct interaction of these solvents with the gold surface. ${ }^{28}$ Cetylpyridinium chloride (CPC) stabilized gold nanoparticles were also used by Ghosh et al. ${ }^{29}$ for determination of chain length of the polar solvent molecules which are capable of forming complexes with gold nanoparticle surface. They also showed a linear relationship between the solvent refractive index and the SPR $\lambda_{\max }$ by drying the metallic particles in toluene and then redispersing in common organic solvents. ${ }^{29}$

In all of these studies, it has been argued that the shift in plasmon peak position is due to the refractive index variations, without any considerations of chemical reactions on the metal particle surfaces. Furthermore, it has only been stated that the shifts are fully reproducible when the refractive index of the solvent is changed in the opposite direction. Similarly, no experimental evidence, confirming the blue shift in the plasmon peak band position due to decrease in refractive index of the medium, has been explicitly presented..$^{4,30}$

In this study, we follow a different approach to alter the refractive index of the medium. Instead of preparing or transferring nanoparticles into different solvents, metal nanoparticles are synthesized in one solvent and then the refractive index of the solution is increased by making serial dilutions with another solvent, having higher (or lower) refractive index value than the original solvent while the wavelength of the peak plasmon wavelength is monitored. Finally, the original solvent is used to bring back the refractive index of the solution in the opposite direction. We believe that this is simple and a much more reliable procedure, which is also more prune to experimental mishaps.

\section{EXPERIMENTAL DETAILS}

All the reagents used were analytical grade. The absorption spectra were recorded using a Varian 5E UV-ViS-NIR spectrophotometer in a $1 \mathrm{~cm}$ quartz cuvette and solvent background was subtracted each time.

(I) Synthesis of colloidal gold nanoparticles in organic phase: Gold nanoparticles capped with tetraocylammonium bromide (TOAB) in toluene were prepared using the biphasic reduction procedure. ${ }^{31,32}$ An aqueous solution of $\mathrm{HAuCl}_{4}(30 \mathrm{~mL}, 3 \mathrm{mM})$ was mixed with a solution of TOAB in toluene $(80 \mathrm{~mL}, 5 \mathrm{mM})$. The two phase mixture was vigorously stirred until all tetrachloroaurate was transferred into the organic layer. A freshly prepared aqueous solution of sodium tetrahydroborate $\left(\mathrm{NaBH}_{4}\right)(25 \mathrm{~mL}$, $40 \mathrm{mM}$ ) was slowly added with vigorous stirring. After further stirring for $3 \mathrm{~h}$, the organic phase was separated.

(II) Synthesis of colloidal gold nanoparticles in aqueous phase: Gold colloids were synthesized following the protocol by Turkevich et al. ${ }^{33,34}$ A $100 \mathrm{~mL}$ solution of $5.0 \times 10^{-4} \mathrm{M} \mathrm{HAuCl}_{4}$ was allowed to boil, at which point $5.0 \mathrm{~mL}$ of $1.0 \%(\mathrm{w} / \mathrm{v})$ sodium citrate was added dropwise with stirring. Following the addition of sodium citrate, the solution began to darken and turn bluish-gray or purple. After approximately $5 \mathrm{~min}$. the reaction was complete and the final color of the solution was a deep wine red.

(III) Synthesis of colloidal silver nanoparticles in aqueous phase: Briefly, colloidal silver was prepared by rapidly adding $1.0 \mathrm{~mL}$ of $0.01 \mathrm{M} \mathrm{AgNO}_{3}$ to $99 \mathrm{~mL}$ of solution containing $1.0 \mathrm{mM} \mathrm{NaBH}_{4}$ and $0.30 \mathrm{mM}$ sodium citrate under vigorous stirring at room temperature. ${ }^{35}$

(IV) Preparation of Iodine solutions $(0.02 \% \mathrm{w} / \mathrm{v})$ : Iodine solution was prepared by dissolving $0.10 \mathrm{~g}$ potassium iodide in $50 \mathrm{~mL}$ of either deionized water or 1-propanol and then $0.010 \mathrm{~g}$ iodine was added, while stirring.

\section{RESULTS AND DISCUSSION}

Different synthesis methods were used in the literature for the preparation of metal nanoparticles and also different capping agents for particle stabilization. ${ }^{31-34,36}$ These procedures usually produce metal nanoparticles of different sizes and shapes. This polydiversity is the major problem for investigating the dependence of the SPR on the refractive index of the medium. $\mathrm{TOA}^{+}$is a positively charged surfactant with four alkane chains and is commonly used as a phase transfer reagent. ${ }^{31,32,37}$ of $\mathrm{AuCl}_{4}^{-}$from water into an organic phase is a well established two phase liquid/liquid synthesis of thiol-derivatized gold nanoparticles. In fact, the four alkane chains in $\mathrm{TOA}^{+}$provide enough binding capacity so that negatively charged water based nanoparticles (free of thiol modification) can also be pulled into toluene phase. In the present study, we were able to prepare $\mathrm{Au}$ nanoparticles capped with TOAB in toluene with a $\lambda_{\max }$ of $522 \mathrm{~nm}$. 
In order to confirm the effect of refractive index of the surrounding medium on the absorption band, first we have studied iodine solutions in water and 1-propanol. When $\mathrm{I}_{2}$ aqueous solution is mixed with KI solution, two absorption peaks appear at 288 and $350 \mathrm{~nm}$, respectively, indicating the formation of triiodide ion. Figure 1(a) shows the variations in the $350 \mathrm{~nm}$ absorption band of triiodide in 1-propanol (1) by diluting first with water (2) and then successively with 1-propanol $(\mathbf{3}, \mathbf{4}$, and 5). A blue shift of absorption band at $350 \mathrm{~nm}$ is measured as expected because the refractive index of the solution decreases by diluting the solution (1) with water. If we further dilute the solution (2) with 1-propanol, this will cause an increase in the refractive index of the surrounding medium which is resulted in red shift as seen from spectra $\mathbf{3}, \mathbf{4}$, and $\mathbf{5}$. Therefore, the absorption band maximum approaches to the initial value (1) with the addition of 1-propanol to Solution 2. In addition, we also performed the opposite of this set of experiment by preparing triiodide solution in water (1) and then dilute with 1-propanol (2) as shown in Figure 1(b). From these experiments, it is concluded that the shifts in the absorption peak positions of the triiodide solution is definitely due to the refractive index variations, and not on chemical reactions occurring with solvent, since the shifts are fully reproducible when refractive index of the solvent is retracted in the opposite direction due to the high stability of the triiodide ion.

The resonance position follows the Mie equation ${ }^{1}$ as

$$
\omega_{1}=\frac{\omega_{\mathrm{p}}}{\sqrt{2 \varepsilon_{\mathrm{med}+1}}}
$$

where $\omega_{\mathrm{p}}$ is the frequency of the analyte and $\varepsilon_{\text {med }}$ is the dielectric constant of the medium which is related to the refractive index of the medium, ${ }^{4} n_{\text {med }}$ by $\varepsilon_{\text {med }}=n_{\text {med }}^{2}$. (a)

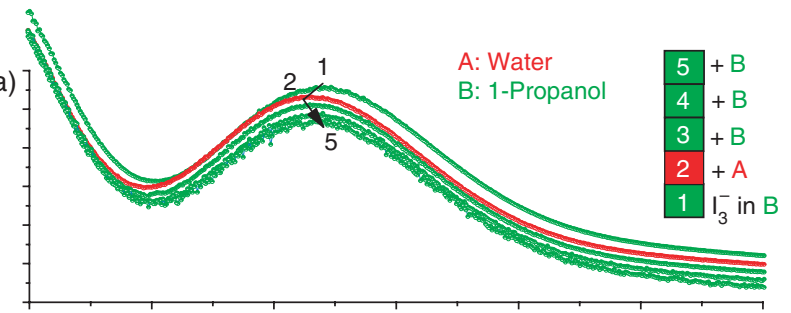

(b)

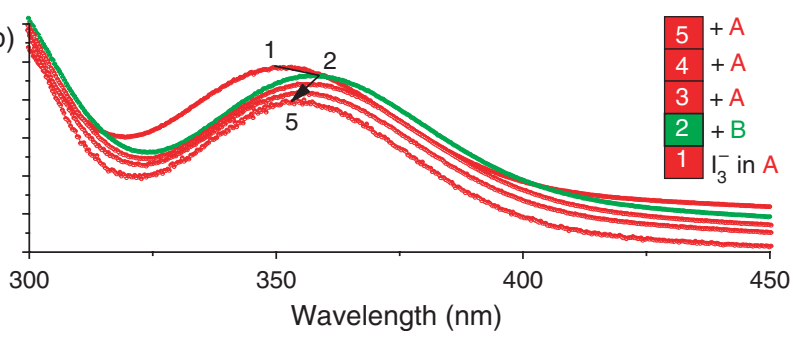

Fig. 1. Absorption band of triiodide (0.02\%w/v) (a) in 1-propanol (1), $2.0 \mathrm{~mL}$ of water added into solution 1 (2), $2.0 \mathrm{~mL} \mathrm{1-propanol} \mathrm{added} \mathrm{into}$ solution 2 (3), 3 (4), and 4 (5); (b) in water (1) $2.0 \mathrm{~mL}$ of 1-propanol added into solution 1 (2), $2.0 \mathrm{~mL}$ water added into solution 2 (3), 3 (4), and $4(\mathbf{5})$. The spectra are normalized for a better comparison.

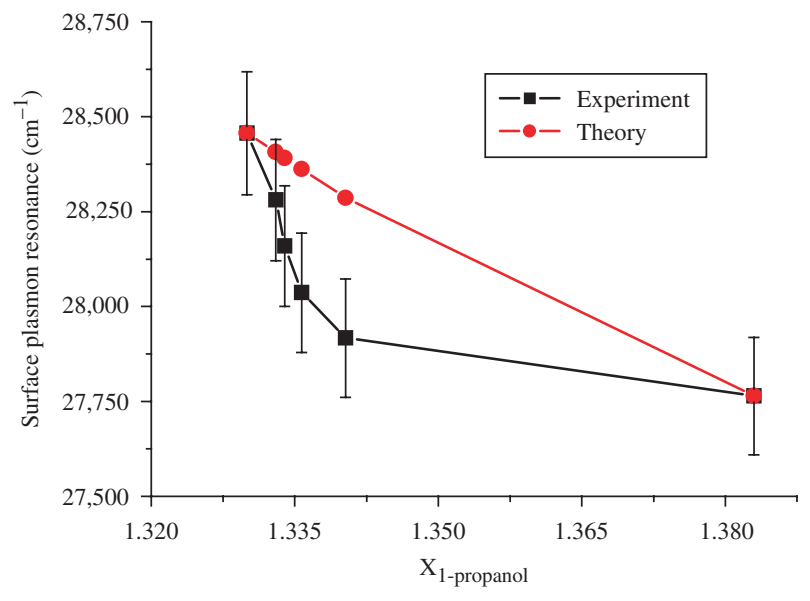

Fig. 2. Plot of peak maximum versus mole fraction of 1-propanol for experimental data and calculated spectra of triodide at $350 \mathrm{~nm}$.

Therefore, a plot of peak energy versus the mole fraction value which is related to refractive index of the medium is linear as shown in Figure 2, along with the experimental results. The experimental shifts are larger, due probably, to the various nonlinearities introduced through mixing (like volume changes, etc.).

The stock solution of TOAB-capped gold nanoparticles $\left(\lambda_{\max }=522 \mathrm{~nm}\right)$ in toluene was diluted using either toluene $\left(n_{\mathrm{D}}=1.494\right)$ or hexane $\left(n_{\mathrm{D}}=1.372\right)$ and the SPR spectra were measured in sequential steps as shown in Figure 3. Solution 1 and Solution 2 were diluted with toluene ( $n_{\mathrm{D}}$ of the solution remains constant) and hexane $\left(n_{\mathrm{D}}\right.$ of the solution decrease), respectively. If we compare SPR bands of $\mathbf{1}$ and $\mathbf{2}$, we expect to see a blue shift since the theory states that the SPR band of nanoparticles shifts toward shorter wavelengths with decreasing refractive index of the solvent. In contrast to the theory,

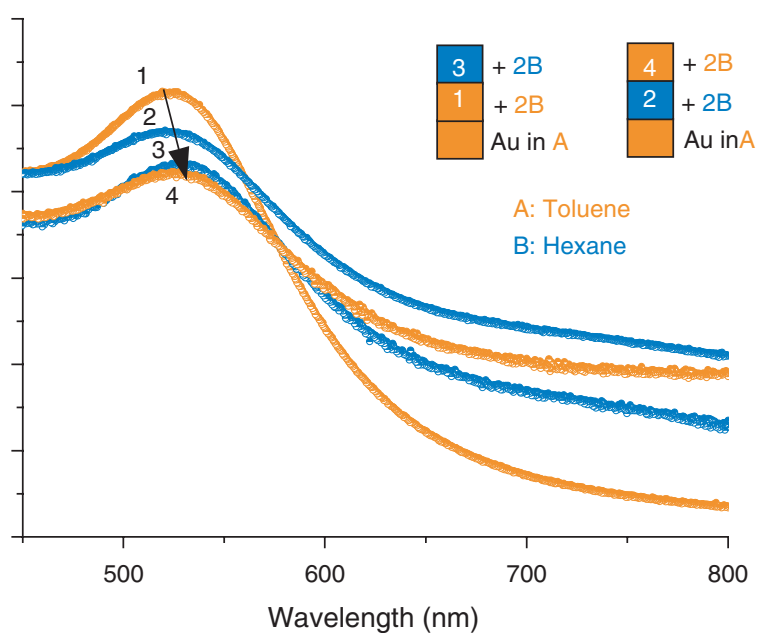

Fig. 3. Surface plasmon absorption band of gold nanoparticles $(1.1 \mathrm{mM})$ in toluene. (1) $10.0 \mathrm{~mL}$ toluene added into $5.0 \mathrm{~mL}$ of stock Au nanoparticles in toluene, (2) $10.0 \mathrm{~mL}$ hexane added into $5.0 \mathrm{~mL}$ of stock $\mathrm{Au}$ nanoparticles in toluene, (3) $10.0 \mathrm{~mL}$ of hexane added into solution 1, (4) $10.0 \mathrm{~mL}$ of toluene added to solution 2 . 


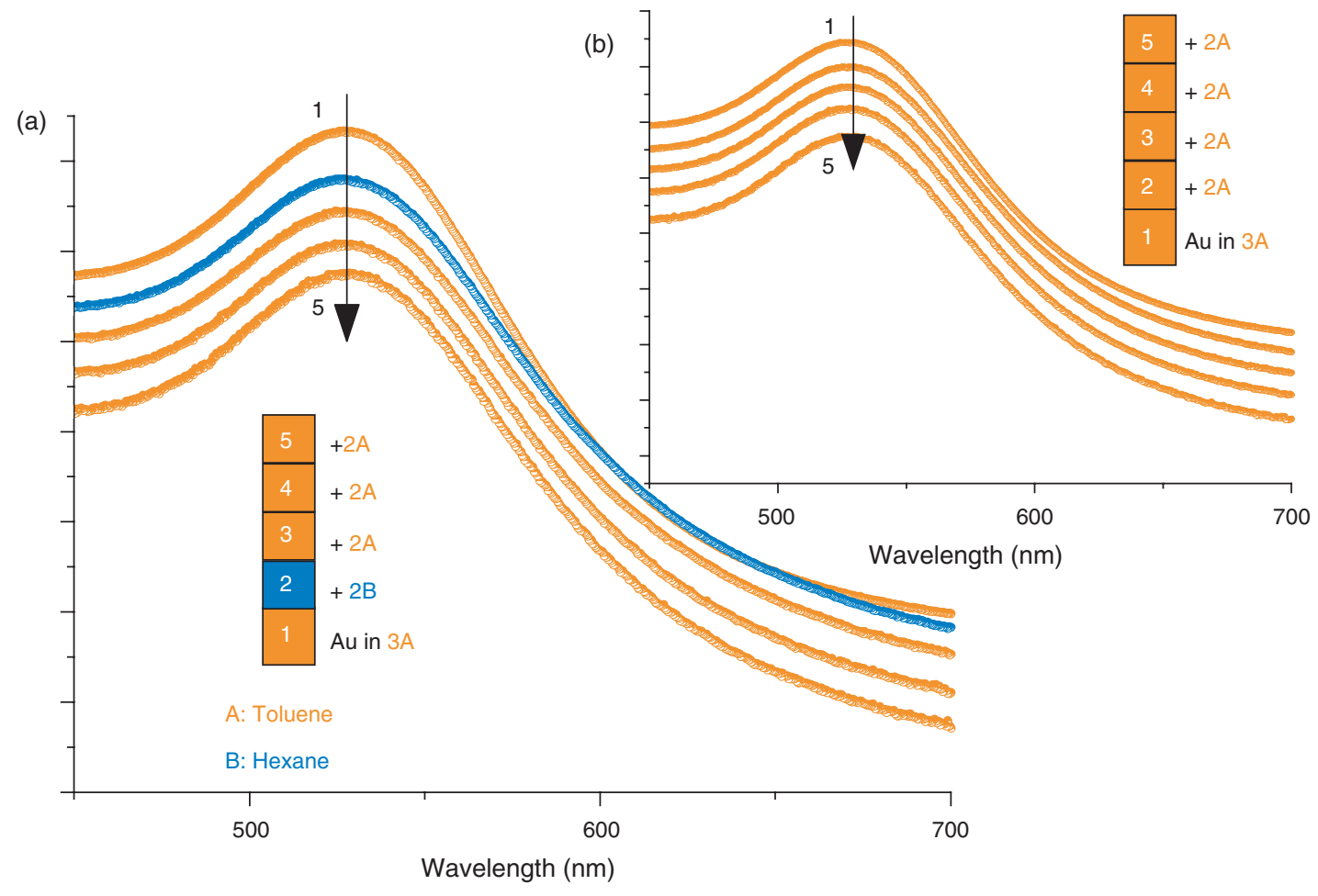

Fig. 4. Surface plasmon absorption band of gold nanoparticles $(1.1 \mathrm{mM})$ in toluene. (a) $3.0 \mathrm{~mL}$ of Au nanoparticles in toluene (1), $2.0 \mathrm{~mL}$ hexane added into solution 1 (2), $2.0 \mathrm{~mL}$ toluene added into solution 2 (3), 3 (4), and 4 (5); (b) sequentially $2.0 \mathrm{~mL}$ toluene added into $3.0 \mathrm{~mL}$ Au nanoparticle solution in toluene in order to show the dilution effect on the position of surface plasmon band. No shift at all is observed.

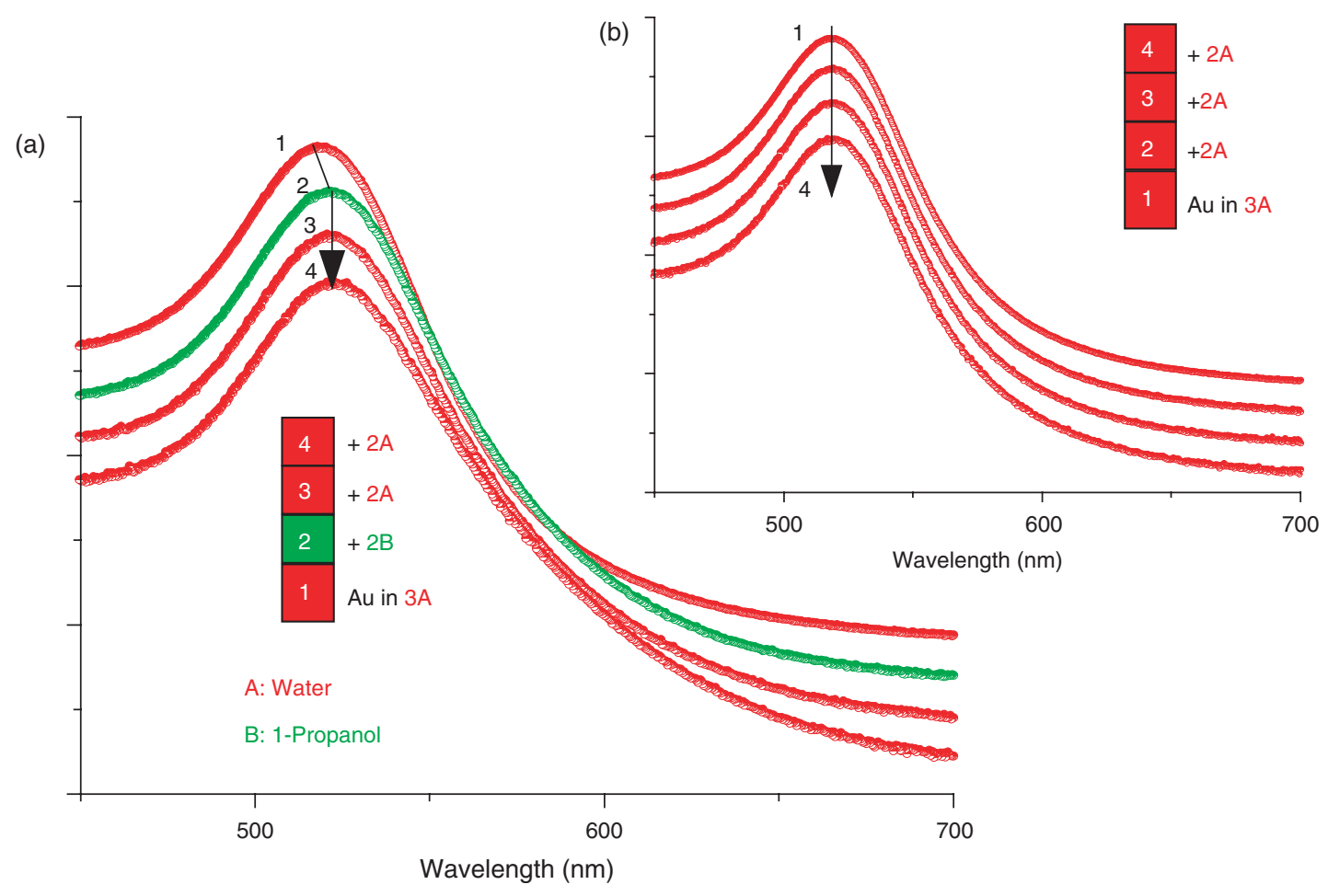

Fig. 5. Surface plasmon absorption band of gold nanoparticles $(0.5 \mathrm{mM})$ in water. (a) $3.0 \mathrm{~mL}$ of Au nanoparticles in water (1), $2.0 \mathrm{~mL}$ of 1-propanol added into solution 1 (2), $2.0 \mathrm{~mL}$ of water added into solution 2 (3) and 3 (4); (b) sequentially $2.0 \mathrm{~mL}$ water added into $3.0 \mathrm{~mL}$ of Au nanoparticle solution in toluene in order to show the dilution effect on the position of surface plasmon band. No shift at all is observed. 


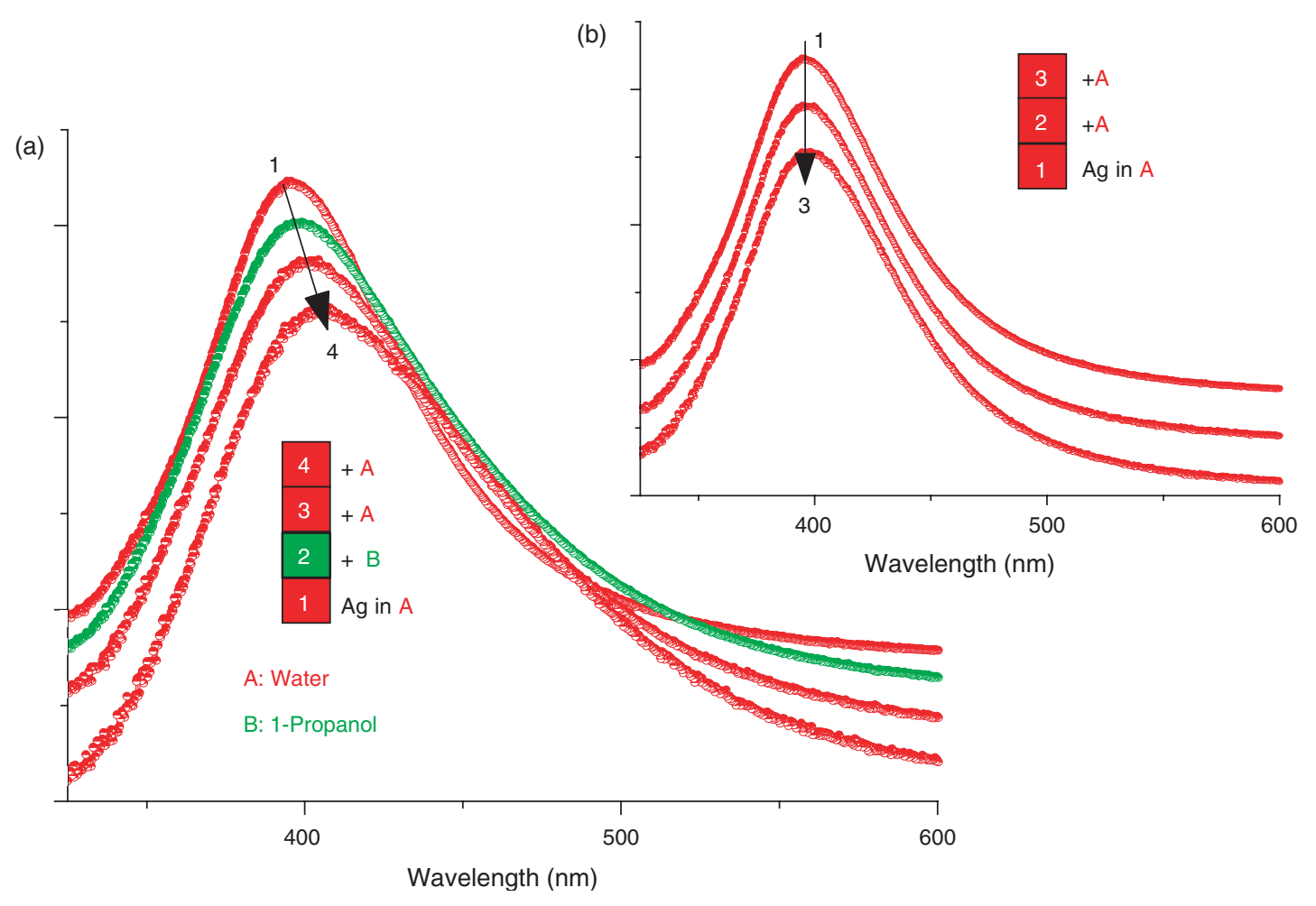

Fig. 6. Surface plasmon absorption band of silver nanoparticles $(0.1 \mathrm{mM})$ in water. (a) $2.0 \mathrm{~mL}$ of $\mathrm{Ag}$ nanoparticles in water (1), $2.0 \mathrm{~mL}$ of 1 -propanol added into solution 1(2), $2.0 \mathrm{~mL}$ water added into solution 2(3) and 3(4); (b) sequentially $2.0 \mathrm{~mL}$ water added into $2.0 \mathrm{~mL}$ of Ag nanoparticle solution in toluene in order to show the dilution effect on the position of surface plasmon band. No shift at all is observed.

a red shift is observed. Further dilution of Solution $\mathbf{1}$ and $\mathbf{2}$ were done by swapping toluene and hexane, thus leading to Solution $\mathbf{3}$ and $\mathbf{4}$ with equal solvent composition. Although refractive indices of Solutions 3 and $\mathbf{4}$ are equal, their SPR band maximum are not at the same energy and the bands increasingly shift to higher wavelengths.

In order to verify the reproducibility of the results shown in Figure 3, gold nanoparticles capped with TOAB in toluene was synthesized with SPR $\lambda_{\max }$ of $529 \mathrm{~nm}$ which is higher compared to previous synthesis of $\mathrm{Au}$ nanoparticles. Two sets of experiments were performed with this new synthesized Au nanoparticles and results are depicted in Figure 4. In the first set, the refractive index of the medium was kept constant but the solution was diluted with toluene in order to ensure that there is no shift in SPR band due to the dilution effect (Fig. 4(b)). This is a strong indication of the stability of TOAB extracted gold nanoparticles in toluene..$^{38}$ In the second set of experiment, the solution was first diluted with hexane (2) ( $n_{\mathrm{D}}$ of the medium decreases) and then serial dilutions were carried out with toluene in order to bring back the refractive index of the solution to the original value $(\mathbf{3}, \mathbf{4}$, and 5) (Fig. 4(a)). In spite of the decrease in refractive index provided with the addition of hexane to the toluene solution (2), no change at all could be observed in the SPR band position even after successive addition of toluene $(\mathbf{3}, \mathbf{4}$, and 5). Previous studies with $4.4 \mathrm{~nm}$ dodecanethiolate-protected $\mathrm{Au}$ nanoparticles $\left(\lambda_{\max }=518 \mathrm{~nm}\right)$ also failed to detect a shift, ${ }^{27}$ however, spectra of $5.2 \mathrm{~nm}$ nanoparticles $\left(\lambda_{\max }=528 \mathrm{~nm}\right)$ reveal an $8-\mathrm{nm}$ red shift as the solvent refractive index is varied from $1.33-1.55 .^{14,26}$

Figure 5(a) shows the SPR spectra for gold nanoparticles in water prepared by Turkevich method with an average diameter of $10 \mathrm{~nm}$, as a function of the solvent refractive index. 1-propanol $\left(n_{\mathrm{D}}=1.383\right)$ was used to change the refractive index of the medium. The plot shows that the SPR band maximum shifts $4 \mathrm{~nm}$ from water to water/1-propanol mixture (Solution 2) which has a higher refractive index value than Au nanoparticles in water. Since Solution 2 has a higher refractive index value than Solution 1, the red shift is an expected result. In order to check if the shift is retractable when the refractive index of the solvent is changed in the opposite direction, Solution 2 is diluted with water twice (Solution 3 and 4). However, no shift is observed towards lower wavelengths. Additionally, no shift at all is observed due to dilution of the nanoparticle solution with water. (Fig. 5(b))

Similar to the gold nanoparticles in water, silver nanoparticles also exhibit a narrow absorption band $\left(\lambda_{\max }=395 \mathrm{~nm}\right)$ which can be recognized in the spectra of 1 in Figure 6(a). Spectral position, halfwidth and intensity of the band also depend on the size and the shape of the silver nanoparticles. Figure 6(a) illustrates the response of the SPR band of silver nanoparticles to changes in the solvent refractive index of the medium after diluting the aqueous solution of silver nanoparticles (1) with 1-propanol (2). 
A similar red shift is observed as in the case of gold nanoparticles (Fig. 5(a)) except for the successive addition of water to Solution 2 (Fig. 6(a)) caused a continued gradual red shift of SPR band maximum. Actually, if the shift was purely due to the refractive index variation, then SPR band would have been shifted towards lower wavelengths with the decrease in refractive index. One particular feature of the SPR is the dependence of the position and width on the particle size. Thus, the red shift and the increase in the halfwidts of the SPR bands of Solutions 1 to 4 (Fig. 6(a)) are most probably related to changes of the shape and/or size of the particles.

As shown in Figures 3 to 6, the changes in SPR band maximum can not be accounted for by simple refractive index changes because the shifts are not retractable when the refractive index of the solvent is changed in the opposite direction, hinting that the plasmon peak position shifts are possibly due to size changes and/or chemical reactions on the nanoparticle surface but not to the refractive index variations.

\section{CONCLUSIONS}

In this paper, the effect of refractive index of the surrounding medium on the SPR band was examined using a different approach which is based on simple serial dilutions with another solvent. For this purpose, we selected a variety of examples from the well defined nanoparticle model systems in a variety of polar and nonpolar solvents. As a general conclusion from these investigations, we believe that every chemical step (mixing with another solvent, or extraction from one phase to another, etc.) introduces changes to the structure and consequently to the optical properties of nanoparticles, hence the effect of the refractive index of the medium is very difficult, if not impossible, to detect experimentally.

Acknowledgments: This work was supported by TUBITAK (The Scientific and Technical Research Council of Turkey) through the Grant No: 106T104.

\section{References and Notes}

1. U. Kreibig and M. Vollmer, Optical Properties of Metal Clusters, Springer, Berlin (1995).

2. N. K. Grady, N. J. Halas, and P. Nordlander, Chem. Phys. Lett. 399, 167 (2004).

3. J. C. Hulteen, D. A. Treichel, M. T. Smith, M. L. Duval, T. R. Jensen, and R. P. Van Duyne, J. Phys. Chem. B 103, 3854 (1999).

4. T. R. Jensen, M. L. Duval, K. L. Kelly, A. A. Lazarides, G. C. Schatz, and R. P. Van Duyne, J. Phys. Chem. B 103, 9846 (1999).

5. D. V. Leff, P. C. Ohara, J. R. Heath, and W. M. Gelbart, J. Phys. Chem. 99, 7036 (1995).
6. C. L. Haynes and R. P. Van Duyne, J. Phys. Chem. B 105, 5599 (2001).

7. C. L. Haynes, A. D. McFarland, L. L. Zhao, R. P. Van Duyne, G. C. Schatz, L. Gunnarsson, J. Prikulis, B. Kasemo, and M. Kall, J. Phys. Chem. B 107, 7337 (2003).

8. W. Fritzsche, H. Porwol, A. Wiegand, S. Bornmann, and J. M. Kohler, Nanostructured Materials 10, 89 (1998).

9. M. Quinten, A. Heilmann, and A. Kiesow, Appl. Phys. A 68, 707 (1999).

10. A. Heilmann, A. Kiesow, M. Gruner, and U. Kreibig, Thin Solid Films 344, 175 (1999).

11. L. B. Scaffardi and J. O. Tocho, Nanotechnology 17, 1309 (2006).

12. L. Yang, G. H. Li, J. G. Zhang, L. D. Zhang, Y. L. Liu, and Q. M. Wang, Appl. Phys. Lett. 78, 102 (2001).

13. M. Quinten, Appl. Phys. B 67, 101 (1998).

14. P. Mulvaney, Langmuir 12, 788 (1996).

15. M. D. Malinsky, K. L. Kelly, G. C. Schatz, and R. P. Van Duyne, J. Am. Chem. Soc. 123, 1471 (2001).

16. C. G. Wang, Z. F. Ma, and Z. M. Su, Nanotechnology 17, 1819 (2006).

17. F. Tam, C. Moran, and N. Halas, J. Phys. Chem. B 108, 17290 (2004).

18. U. Kreibig, M. Gartz, A. Hilger, and R. Neuendorf, Nanostructured Materials 11, 1335 (1999).

19. H. J. Lee, D. Nedelkov, and R. M. Corn, Anal. Chem. 78, 6504 (2006).

20. Y. A. Li, A. W. Wark, H. J. Lee, and R. M. Corn, Anal. Chem. 78, 3158 (2006)

21. A. Dienst, A. Grunow, M. Unruh, B. Rabausch, J. E. Nor, J. W. U. Fries, and C. Gottstein, J. Natl. Cancer Inst. 97, 733 (2005).

22. A. Okazaki, E. Shoji-Hosaka, K. Nakamura, M. Wakitani, K. Uchida, S. Kakita, K. Tsumoto, I. Kumagai, and K. Shitara, J. Mol. Biol. 336, 1239 (2004).

23. A. J. Haes, S. L. Zou, G. C. Schatz, and R. P. Van Duyne, J. Phys. Chem. B 108, 6961 (2004)

24. P. Hanarp, M. Kall, and D. S. Sutherland, J. Phys. Chem. B 107, 5768 (2003).

25. S. Underwood and P. Mulvaney, Langmuir 10, 3427 (1994).

26. A. C. Templeton, J. J. Pietron, R. W. Murray, and P. Mulvaney, J. Phys. Chem. B 104, 564 (2000).

27. M. J. Hostetler, J. E. Wingate, C. J. Zhong, J. E. Harris, R. W. Vachet, M. R. Clark, J. D. Londono, S. J. Green, J. J. Stokes, G. D. Wignall, G. L. Glish, M. D. Porter, N. D. Evans, and R. W. Murray, Langmuir 14, 17 (1998).

28. K. G. Thomas, J. Zajicek, and P. V. Kamat, Langmuir 18, 3722 (2002).

29. S. K. Ghosh, S. Kundu, S. Nath, and T. Pal, Appl. Spectrosc. 59, 844 (2005)

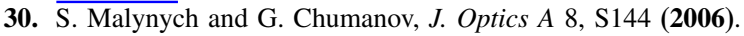

31. M. Brust, J. Fink, D. Bethell, D. J. Schiffrin, and C. Kiely, J. Chem. Soc. Chem. Commun. 16, 1655 (1995).

32. M. Brust, M. Walker, D. Bethell, D. J. Schiffrin, and R. Whyman, J. Chem. Soc. Chem. Commun. 7, 801 (1994).

33. B. V. Enustun and J. Turkevich, J. Am. Chem. Soc. 85, 3317 (1963).

34. J. Turkevich, P. L. Stevenson, and J. Hillier, Discuss. Faraday Soc. 11, 55 (1951).

35. T. Ung, L. M. Liz-Marzan, and P. Mulvaney, J. Phys. Chem. B 103, 6770 (1999).

36. A. Henglein and D. Meisel, Langmuir 14, 7392 (1998).

37. J. Fink, C. J. Kiely, D. Bethell, and D. J. Schiffrin, Chem. Mater. 10, 922 (1998).

38. W. L. Cheng and E. Wang, J. Phys. Chem. B 108, 24 (2004).

Received: 29 December 2006. Accepted: 28 April 2007. 